\title{
GW23-e2182 PATIENTS WITH SINGLE CORONARY ARTERY MALFORMATIONS AND ACUTE INFERIOR MYOCARDIAL INFARCTION UNDERGOING EMERGENCY PCI: 1 CASE
}

doi:10.1136/heartjnl-2012-302920l.36

Xie Dongyang, Liu Hairong. Department of Cardiology, Affiliated Hospital of Gannan Medical College, JiangXi, GanZhou

\section{Objectives}

1. Clinical Data: Patient, male, 48 years old, chest pain $5 \mathrm{~h}$, aggravation $4 \mathrm{~h}$, was admitted to hospital on 9 February 2012.

\section{Methods}

2. Discussion: Single coronary artery malformation is rare, and it is origin from single opening to supply with entire heart blood.

Results This patient appeared chest pain and chest tightness aggravation in short time, companion with hypotension, alternation accelerated ventricular arrhythmia with sinus bradycardia, ST segment re-elevation, the necrotising $\mathrm{Q}$ wave deeper in intraoperative reperfusion therapy. Reperfusion therapy made ischemicinjured-myocardium worse, aggravated myocardial cells injury, even necrosis. In preoperative and postoperative period, daily ECG showed that $\mathrm{ORS}$ waves had obvious variation. In preoperative day showed $Q R$ waves $(R>Q)$, intraoperative period showed $O S$ waves and $\mathrm{R}$ wave disappeared. The third postoperative day showed $\mathrm{Or}$ waves, the fourth day showed $O R$ waves $(R<Q)$, the fifth day showed $Q R$ waves $(R>Q)$. It explained that reperfusion injury induced part myocardial stunning, and the stunned myocardium recuperated activity within a few days.

Conclusions Experiences: First, if conventional method of doing CAG failed to find the coronary openings, abnormal coronary openings should be considered. Second, single coronary artery malformations is rare, down opening and acute occlusion of RCA increase the difficulty of CAG and PCI. Third, when do emergency PCI, we should pay special attention to ischaemia-reperfusion injury. $\mathrm{R}$ Reperfusion is a double-edged sword, it can provide timely help for part ischemic myocardium, also can make part ischaemic-injuredmyocardium worse, aggravated myocardial cells injury, even necrosis. Myocardial stunning may be the main performance of aggravated injury, and stunned myocardium recuperated activity within a few days. In postoperative days, daily ECG may be found ORS waves dynamic variation. 